Trojanowska Paulina, Chrościńska-Krawczyk Magdalena, Trojanowska Alina, Tywanek Ewa, Wronecki Jakub, Luczyk Robert. The role of selected mechanisms of innate immunity in the pathogenesis of diabetes. Journal of Education, Health and Sport. 2021;11(9):544-549. eISSN 2391-8306. DOI http://dx.doi.org/10.12775/JEHS.2021.11.09.071

https://apcz.umk.pl/JEHS/article/view/JEHS.2021.11.09.071

https://zenodo.org/record/5529296

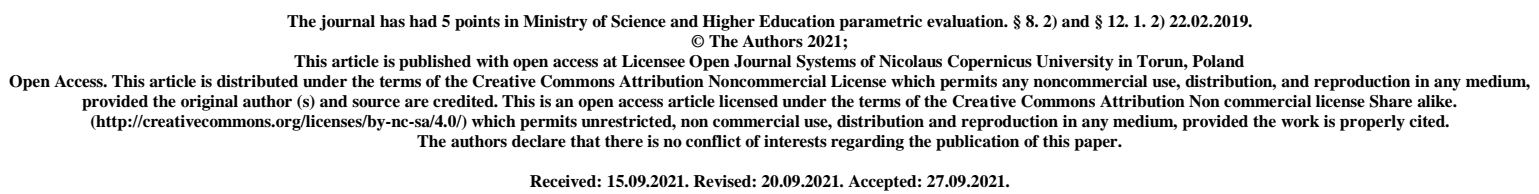

\title{
The role of selected mechanisms of innate immunity in the pathogenesis of
} diabetes

\section{Paulina Trojanowska, Magdalena Chrościńska-Krawczyk, Alina Trojanowska, Ewa Tywanek, Jakub Wronecki, Robert Jan Luczyk}

1. Paulina Trojanowska, trojanowska_paulina@wp.pl, ORCID: 0000-0002-5289-6473, Department of Paediatric Neurology and Department of Endocrinology, Diabetology and Metabolic Diseases, Medical University of Lublin, Poland

2. Magdalena Chrościńska-Krawczyk, magdalenachk@wp.pl, ORCID: 0000-0001-81216580, Department of Paediatric Neurology, Medical University of Lublin, Poland

3. Alina Trojanowska, trojanowska-a@wp.pl, ORCID: 0000-0002-4021-7509, Department of Paediatrics and Paediatric Nursing, Faculty of Health Sciences, Medical University of Lublin

4. Ewa Tywanek, ewa.tywanek@gmail.com, ORCID: 0000-0002-2311-994X, Chair of Internal Medicine and Department of Internal Medicine in Nursing, Medical University of Lublin, Poland; Department of Endocrinology with Department of Nuclear Medicine, Center of Oncology of the Lublin Region St. Jana z Dukli in Lublin, Jaczewskiego 7, 20-090 Lublin 5. Jakub Wronecki, wronecki.jakub@gmail.com, ORCID: 0000-0001-8664-7072, Chair and Department of Gastroenterology with Endoscopic Unit, Medical University of Lublin 6. Robert Jan Łuczyk, luczykrobert@op.pl ORCID: 0000-0003-0889-6326, Internal Medicine and Department of Internal Medicine in Nursing, Medical University of Lublin, Poland 


\begin{abstract}
Understanding the important role of the non-specific immune response in protecting the body against the development of numerous diseases has become partially possible after the discovery of several classes of pattern recognition receptors (PRR), such as Toll-like or NODlike receptors. A group of cytoplasmic proteins called the inflammasome, which detect PAMP and DAMP through the PRR receptors, is able to activate pro-inflammatory cytokines and trigger an acute inflammatory reaction both in the extracellular and intracellular space. Lowgrade systemic and local inflammation contributes to the development and progression of various conditions, including autoimmune and metabolic diseases, such as diabetes, metabolic syndrome and atherosclerosis, which until recently were not even considered inflammatory diseases. This review will discuss the role of innate immunity in the development of type 1 and type 2 diabetes, focusing on the role of specific innate immunity receptors and insulin resistance involved in these diseases pathogenesis.
\end{abstract}

Key words: type 1 diabetes, type 2 diabetes, innate immunity, insulin resistance

\title{
Introduction
}

The growing epidemic of obesity and insulin resistance has made the incidence of both type 1 (T1D) and type 2 (T2D) diabetes the highest ever. The World Health Organization (WHO) estimates that approximately 422 million people worldwide suffer from diabetes, and 1.6 million deaths are directly related to this disease. It is essential that the incidence of T1D in people with the so-called "high risk" HLA haplotypes (predisposing to the development of this condition) has remained stable over the last twenty years, while the incidence of the "medium", "low" and "very low risk" haplotypes has increased significantly [1]. Importantly, the phenotype of a patient with T1D has changed in recent years, showing a tendency towards obesity and insulin resistance and often meeting the full criteria of T2D diagnosis. Moreover, obesity-related insulin resistance not only precedes clinical disease, but is also the strongest predictor of T1D, in addition to specific haplotypes of the HLA system. The emergence of almost ubiquitous obesity has extended the once strict criteria of T1D and T2D to such an extent that obesity-related insulin resistance has become a significant risk factor for the development of both types of diabetes. In turn, chronic inflammation, which occurs in both type 1 and type 2 diabetics, contributes to the development of insulin resistance [1]. Although the specific immune response plays an important role in the pathogenesis of T1D, the identification of new innate immune molecules has led many scientists to investigate their role in the long-term inflammatory process in both T1D and T2D [2]. It has been shown that the accumulation of activated cells of innate immunity in tissues with high metabolic activity, including adipose tissue, causes the release of inflammatory mediators, in particular the proinflammatory cytokines IL-1 $\beta$ and TNF $\alpha$, which contributes to the development of systemic and local low-grade inflammation, leading to insulin resistance and, as a consequence, destruction of pancreatic $\beta$ cells $[1,4]$. 
While most of the research to date on the pathogenesis of diabetes has focused on the involvement of specific immunity, observations of development, course and treatment of this condition in recent years have led to a greater focus on the role of innate immunity instead. Numerous studies support the hypothesis that the development of diabetes is caused by an increase in innate immune activity, combined with disruptions in central and peripheral tolerance mechanisms, which lead to the expansion of autoreactive $\mathrm{T}$ lymphocytes. It was noticed that monotherapies aimed at stopping specific immune response did not permanently inhibit the disease, while recent preclinical studies, that applied combination therapies targeting both innate and acquired immunity showed greater efficacy. Additionally, the rise in T1D incidence in recent decades is too rapid to be caused solely by genetic alteration; this is partly supported by the observation that individuals with "low risk" HLA haplotypes currently have the highest rate of increase in T1D incidence. These epidemiological changes suggest the presence of environmental alteration that enhance autoimmunity, possibly by dysregulating innate immunity $[1,3]$.

\section{Damage Associated Molecular Patterns (DAMP) and Pattern Recognition Receptors (PRR)}

Unlike a specific immune response, the non-specific response allows the body to detect and destroy most microbial effects within minutes or hours, using defence mechanisms that are independent of clonal selection. These mechanisms include Pathogen Associated Molecular Patterns (PAMP) detection via Pattern Recognition Receptors (PRR). In addition to reacting to antigens of microorganisms, the immune system has developed the ability to detect endogenous threats by recognizing the so-called Danger Associated Molecular Patterns (DAMP). DAMP, or alarmins, are endogenous molecules released as a result of cell damage or death, which, signalling a threat, activate the mechanisms of non-specific immunity through PRR [2]. Alarmins can be compounds such as small molecules located intracellularly and released by necrotic cells (ATP, DNA, RNA, uric acid crystals), glycosylated proteins, or misplaced proteins / antigens. Typical PRR responsible for the detection of PAMP / DAMP are represented by, among others, Toll-like receptors (TLR) or NOD-like receptors (NLR) [2, 4]. In diabetes, insulin-dependent cells may be damaged due to hypoglycaemia, while insulinindependent cells struggle with chronic hyperglycaemia. The metabolic imbalance, that resulted from it, can generate both intracellular (e.g. accumulation of proteins with abnormal spatial structure) and extracellular (e.g. advanced glycation end-products) DAMP. Intracellular DAMP can be detected by PRR in the cytosol and induce an autonomous cellular inflammatory response, while extracellular DAMP are often detected by PRR on the cell surface and induce an inflammatory response at the tissue or even systemic level. The physiological role of the immune response is to remove DAMP and maintain homeostasis [4].

It has been reported that a fundamental role in initiating an autoimmune inflammatory response in T1D is carried out by detection by innate immune receptors such as Toll-like receptors (TLR) of DAMP, which are released from apoptotic $\beta$ cells in the pancreas, suggesting that the non-specific immune response is crucial for the development of this disease. What concerns T2D, it has long been recognized as a metabolic disorder that is pathogenetically distinct from T1D. However, recent studies have found that chronic inflammation contributes significantly to the origin of T2D. 
While it is not yet fully understood the activity of which innate immunity receptors are involved in the development of T2D, usually associated with obesity, numerous studies have shown an important role of TLR4 or TLR2, as well as family of NOD-like receptors, such as NLR family pyrin domain containing 3 (NLRP3). NLPR3 is an inflammasome, an intracellular complex located in the cytosol, which is an element of non-specific immunity, responsible for the detection of DAMP, PAMP and the production of pro-inflammatory cytokines. NLRP3 plays a key role in the maturation and release of IL-1 $\beta$. Animal studies have shown a positive correlation between pro-IL-1 $\beta$ or NLRP 3 mRNA expression and body weight. Some studies have shown that inhibition of the NLRP3 inflammasome activation may be a component of the therapeutic effect of some antidiabetic drugs, such as glyburide or metformin, although the molecular and cellular mechanisms of this phenomenon are not fully understood. Instead, the molecular mechanism of the NLRP3 inflammasome activation is well known: after contact with DAMP or PAMP, NLRP3 self-oligomerizes and then binds to the ASC (Apoptosis-associated speck-like protein containing a CARD), activating caspase- 1 and therefore a maturation of IL-1 $\beta$ from pro-IL-1 $\beta$. However, the steps that precede the oligomerization of NLRP3 themselves are not completely clear, while the reactions taking place within lysosomes and mitochondria are taken into account [5].

\section{Insulin resistance}

Insulin is a polypeptide that has pleiotropic effects on almost every type of tissue in the organism. This hormone is involved in the regulation of basic cellular functions such as growth, proliferation and apoptosis. Since adipose tissue, liver and skeletal muscle are the primary targets of the metabolic activity of insulin, they are recognized as the leading determinants of insulin resistance. The pancreas, on the other hand, is also a target of insulin resistance, albeit indirectly. Due to impairment of peripheral insulin activity and with increasing insulin resistance, blood glucose levels gradually increase, which in turn leads to compensatory and progressive hyperinsulinemia. The consequence of this pathology is an increase of oxidative stress in $\beta$ cells, the final depletion of $\beta$ cells and the development of diabetes mellitus. This hypothesis is supported, inter alia, by the fact that many genes that play their role in the pathogenesis of T2D (e.g., INS, KCNJ11, and TCF7L2) are expressed not in insulin target tissues, but in the pancreatic islets themselves. This result of insulin resistance has always been viewed as the end stage of T2D; however, there is evidence that this process works parallelly with the progressive destruction of $\beta$-cell mass by immune mechanisms in all stages of diabetes.

In particular, three mechanisms link insulin resistance to progressive $\beta$-cell dysfunction and indirect immune attack: glucotoxicity, lipotoxicity, and inflammation. Increased levels of glucose and lipids, especially saturated fatty acids, which are characteristic of insulin resistance, have a synergistic effect at the $\beta$-cell level, stimulating a parallel increase in FAS receptor expression and activating NK cell ligands (e.g. RAE-1, NKp46 ligand), reactive oxygen species and stress of the endoplasmic reticulum (ER) leading to IL-1 $\beta$ secretion and apoptosis. Importantly, IL-1 $\beta$ is a known mediator of $\beta$ cell dysfunction and death, and this process is exacerbated by the action of other inflammatory mediators, such as TNF $\alpha$ and IFN $\gamma$, which concentrations are elevated in insulin resistance [1,6]. Diabetics have elevated baseline levels of innate inflammation characterized by an increase in the secretion of IL-1. 
Among pro-inflammatory cytokines, IL-1 has been one of the most frequently chosen targets for the treatment of T1D in several large clinical trials [6]. Indeed, $\beta$ cells are extremely susceptible to IL-1 $\beta$, because they have a higher concentration of IL-1R1 receptors than any other cell in the system. Activation of IL-1R1 results in the stimulation of NF- $\kappa B$, MAPK, PKC $\delta$ and JNK signalling pathways, leading to the direct promotion of apoptosis and upregulation of FAS receptors, as well as inhibition of insulin signalling pathways, which are essential for optimal $\beta$-cell function. Moreover, the activity of IL-1 $\beta$ leads to the production of other pro-inflammatory mediators that act in an autocrine / paracrine manner in $\beta$-cells and local innate immunity cells to enhance these effects [1].

The consequence of these processes is the formation of a microenvironment of pancreatic islets full of damaged $\beta$-cell mass, antigenic remains of $\beta$-cells and cells of the local innate inflammatory response. The response to such damage to the pancreas is to trigger a lymphocyte-stimulated autoimmune attack on the remaining $\beta$-cell reserve. Whether this attack occurs early in the disease, when a significant proportion of the $\beta$-cell mass remains intact (typically in T1D), or later, when a significant proportion of the $\beta$-cell mass has been degraded by long-term insulin resistance (typically in T2D), the end mechanisms of $\beta$-cell failure does not differ. As such, the combination of insulin resistance with exhaustion, direct toxicity and autoimmune $\beta$-cell destruction results in the scale of obesity epidemic leading to historically high levels of T1D and T2D [1].

\section{Macrophages}

Numerous clinical studies emphasize the role of macrophages in the pathogenesis of obesity, insulin resistance and diabetes [7]. There are two macrophage subtypes in adipose tissue: the pro-inflammatory macrophage type "M1" and the anti-inflammatory macrophage type "M2", which is characterized by the secretion of anti-inflammatory cytokines such as IL4 and IL-10. In obese patients, macrophages showed profound pro-inflammatory (M1) polarization under the influence of local environmental factors in adipose tissue and are therefore considered to be the main source of pro-inflammatory cytokines and chemokines. By penetrating into the expanding adipose tissue, phagocytes induce local inflammation in it, which may explain the relationship between obesity and insulin resistance. These immune cells also secrete chemokines to recruit the next wave of incoming monocytes. IL-4-induced loss of macrophage-induced peroxisome proliferator-activated receptor- $\gamma$ (PPAR- $\gamma$ ) expression may also result in impaired insulin sensitivity in the liver and muscles. Weakened PPAR $\gamma$ expression in bone marrow cells reduces the activation of anti-inflammatory macrophages (M2 type), thus playing a key role in the development of diet-induced obesity, insulin resistance and glucose intolerance [7].

Additionally, pancreatic islets isolated from type 1 and type 2 diabetics are characterized by an abnormally high representation of macrophages. This infiltration of macrophages into the pancreatic islets is responsible for the progress of peripheral insulin resistance and precedes the development of a targeted immune response in the pancreas. Moreover, increasing levels of M1 macrophages correlate with increased expression of classical activation markers including IL- $1 \beta$ and $\mathrm{TNF} \alpha$, which suppress insulin secretion and signalling in pancreatic islets and peripheral tissues, respectively, and are involved in apoptosis and $\beta$-cell destruction. 
Macrophages also synthesize and release IL-12, facilitating the conversion of naive T cells to mature cytotoxic T cells (CTL), a known factor in the pathophysiology of T1D. They also have the ability to produce reactive oxygen species (ROS) in injured pancreas, which leads to further cell apoptosis and acceleration of T1D development. Therefore, inhibition of macrophage recruitment effectively stops the development and progression of T1D and T2D $[1,6,8]$.

\section{Conclusions}

In recent decades, many different publications on the pathogenesis of diabetes have appeared, on the basis of which the current traditional dualistic concept of type 1 and type 2 diabetes is no longer relevant. On the contrary, obesity, lack of physical activity, poor eating habits and other environmental conditions of the modern world have made inflammatory insulin resistance a major factor in the development and progression of diabetes. Recognizing inflammation-induced insulin resistance as a major determinant of diabetes has important therapeutic implications. Indeed, therapeutic approaches originally limited to type 2 diabetes, for example - weight loss, exercise, and any measures to improve tissue insulin sensitivity have shown remarkable efficacy in preventing / slowing the development of type 1 diabetes, and also underpinning the importance of integrated treatment of diabetes. Thanks to these approaches, therapies have not only stopped the continuous destruction of $\beta$ cells, but also, for the first time, actually rebuild their mass using the natural regenerative capacity of $\beta$ cells [1].

\section{Bibliography:}

[1] Odegaard JI, Chawla A. Connecting Type 1 and Type 2 Diabetes through Innate Immunity. Cold Spring Harb Perspect Med. 2012; 2(3).

[2] Wada J, Makino H. Innate immunity, pattern recognition receptors, and inflammasomes in diabetes and diabetic nephropathy. Nat Rev Nephrol. 2016 Jan;12(1):13-26.

[3] Cabrera SM, Henschel AM, Hessnera MJ. Innate inflammation in Type 1 diabetes. Trans1 Res. 2016 Jan; 167(1): 214-227.

[4] Xu H, Chen M. Diabetic retinopathy and dysregulated innate immunity. Vision Research. 2017. Volume 139, Pages 39-46.

[5] Myung-Shik L. Role of Innate Immunity in the Pathogenesis of Type 1 and Type 2 Diabetes. J Korean Med Sci. 2014 Aug; 29(8): 1038-1041.

[6] Gao S, Wolanyk N, Chen Y, Jia S, Hessner MJ, Wang X. Investigation of coordination and order in transcription regulation of innate and adaptive immunity genes in type 1 diabetes. BMC Med Genomics. 2017; 10:7.

[7] Zhou T, Hu Z, Yang S, Sun L, Yu Z, Wang G. Role of Adaptive and Innate Immunity in Type 2 Diabetes Mellitus. J Diabetes Res. 2018; 2018: 7457269.

[8] Sun L, Xi S, He G, Li Z, Gang X, Sun C, Guo W, Wang G. Two to Tango: Dialogue between Adaptive and Innate Immunity in Type 1 Diabetes. J Diabetes Res. 2020; 2020: 4106518. 